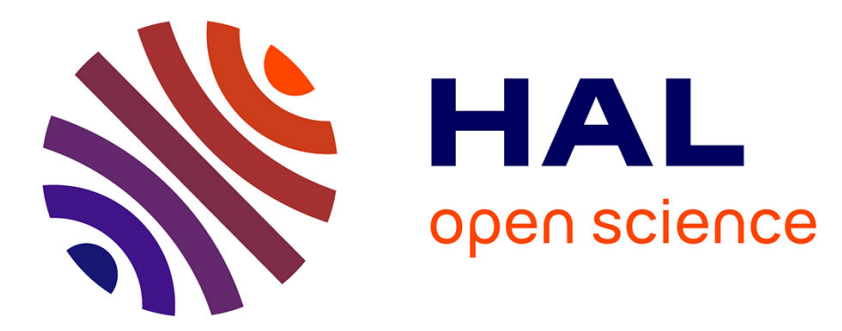

\title{
Persistent coordination patterns in a complex task after 10 years delay
}

Déborah Nourrit-Lucas, Gregory Zelic, Thibault Deschamps, Michael Hilpron, Didier Delignières

\section{- To cite this version:}

Déborah Nourrit-Lucas, Gregory Zelic, Thibault Deschamps, Michael Hilpron, Didier Delignières. Persistent coordination patterns in a complex task after 10 years delay. Human Movement Science, 2014, 32, pp.1365-1378. 10.1016/j.humov.2013.07.005 . hal-00915740

\author{
HAL Id: hal-00915740 \\ https://hal.science/hal-00915740
}

Submitted on 10 Dec 2013

HAL is a multi-disciplinary open access archive for the deposit and dissemination of scientific research documents, whether they are published or not. The documents may come from teaching and research institutions in France or abroad, or from public or private research centers.
L'archive ouverte pluridisciplinaire HAL, est destinée au dépôt et à la diffusion de documents scientifiques de niveau recherche, publiés ou non, émanant des établissements d'enseignement et de recherche français ou étrangers, des laboratoires publics ou privés. 
Title

Persistent coordination patterns in a complex task after 10 years delay ${ }^{1}$.

\section{Authors names and affiliations}

Déborah Nourrit-Lucas $^{\mathrm{a}^{*}}$, Grégory Zelic ${ }^{\mathrm{b}}$, Thibault Deschamps ${ }^{\mathrm{c}}$, Michael Hilpron ${ }^{\mathrm{d}}$ \& Didier Delignières $^{b}$

a* UMR 7349 MAPMO, UFR Sciences, Orléans University, France, deborah.lucas@univorleans.fr

b EA 2991 Movement to Health, UFR STAPS, Montpellier University 1, France, gregory.zelic@univ-montp1.fr ; didier.delignieres@univ-montp1.fr

${ }^{\text {c }}$ EA 4334 Motricité, Interaction, Performance, UFR STAPS, Nantes University, France, thibault.deschamps@univ-nantes.fr

d EA 4248 AMAPP, UFR STAPS, Orléans University, France

\footnotetext{
${ }^{1}$ Subtitle : How validate the old saying "Once you have learned how to ride a bicycle, you never forget!".

${ }^{1 *}$ Corresponding author: D. Nourrit-Lucas, UMR. 7349 "Mathématiques-Analyse, Probabilités, ModélisationOrléans", Département de Mathématiques, Université d'Orléans, Rue de Chartres, B.P. 6759-F-45067 Orléans cedex 2 France Tel: +332384946 81. Fax: +3323841 72 05. E-mail: deborah.lucas@univ-orleans.fr
} 


\section{Abstract}

Motor learning studies have for a long time focus on performance variables (in terms of speed or accuracy) for assessing learning, transfer and retention of motor skills. We argue, however, that learning essentially lies on changes in coordination variables (in terms of qualitative organization of behavior) and that relevant test for assessing the effectiveness of learning and retention should consider these variables. The aim of this experiment was to test the retention of a complex motor skill, after a long term delay. 10 years ago, five participants were involved in an experiment during which they practiced for 39 sessions of ten 1-min trials on a ski-simulator. All participants volunteered for a retention test, ten years after, for one session of ten 1-min trials. Analyses focused on the oscillations of the platform of the simulator. Performance was assessed in terms of amplitude and frequency. Coordination was accounted for by an analysis of dynamical properties of the motion of the platform, and especially the nature of the damping function that was exploited for sustaining the limit cycle dynamics. Results showed a significant decrement in performance variables. In contrast, all participants adopted from the first trial the coordination mode they learned 10 years ago. These results confirm the strong persistence of coordination modes, once acquired and stabilized in the behavioral repertoire. They also support the importance of coordination variables, for a valid assessment of learning and retention.

Key-words: Motor learning, retention, dynamical modeling 


\section{Introduction}

Motor learning is defined as a permanent change in behavior in a specific task, resulting from practice (Schmidt, 1982). This definition emphasizes the importance of retention tests for assessing learning: a change in behavior should be proven to present a certain stability over time, for being considered a valid indication of learning.

Behavior changes, during learning, are not restricted to these long-term and permanent modifications. Changes occur at different levels and following diverse time scales. According to Newell, Liu, \& Gottfried (2001), the evolution of behavior during learning is also affected by transitory changes, as, for example, the warm-up decrement, a systematic decrease in performance that occurs at the beginning of each practice session, with respect to the level of performance reached at the end of the previous session, the alterations of behavior that could occur during a session, due to fatigue and drop in attention, and finally to trial-to-trial fluctuations, generally interpreted as random variability.

However, the nature of the changes observed during learning is dependent on the characteristics of task to be learned, and also on the variables that are used for describing behavior. It seems useful, at this level, to distinguish between two categories of variables, commonly used in motor learning experiments.

Performance variables focus on the outcomes of behavior, with respect to the goal of the task, in terms of speed (reaction time, movement time) or accuracy (absolute and variable errors, etc.). In contrast, coordination variables aim at accounting for the functional organization of behavior. These variables generally describe the spatio-temporal relationships between body parts, or between the body and the environment, in terms of relative phase (Kelso, 1995), or by means of dynamical models capturing the essential features of oscillatory behaviours (Beek \& Beek, 1988). 
In most motor learning experiments, up to the 80s, learning was assessed through performance variables. The first reason was related to the fact that the dominant paradigm, considering motor learning as an optimization of information processing, was primarily interested in problems of speed and accuracy (Abernethy \& Sparrow, 1992). Experimental tasks were generally quite simple (linear positioning, target reaching, etc.), involving a few number of degrees of freedom. More recently, the development of the dynamical systems approach and the focus on coordination, as a property emerging from a complex set of constraints during the performance of the task, have motivated the use of coordination variables (Beek, Peper, \& Stegeman, 1995). Generally, these experiments analyzed learning in more complex tasks, requiring at least the coordination of two body segments (Zanone \& Kelso, 1992; 1997), and often in gross motor skills involving a huge number of degrees of freedom (Vereijken, 1991, Delignières, Nourrit, Sioud, Leroyer, Zattara, \& Micaleff, 1998; Nourrit, Delignières, Caillou, Deschamps, \& Lauriot, 2003).

These two contrasted approaches yielded different conclusions about changes during learning. Experiments focusing on performance variables in simple tasks generally considered learning as the progressive and continuous refinement of information processing. Performance variables were often showed to evolve, during the learning process, following a power law. According to Newell (1991), this power law that was for a long time considered a powerful and universal principle, could represent an artifact due to the simplicity of the tasks, and the nature of the variables used. The author showed that learning in more complex tasks, involving multiple degrees of freedom, presented in contrast a discontinuous character, marked by abrupt changes in behavior during the course of learning.

Another important point is that motor learning experiments could strongly differ, in terms of scientific aims and practical interests. At least two categories could be distinguished: in the first category experiments seek at understanding the process of acquisition of a novel motor 
skill: participants are facing a completely novel task, and they have to build a new behavioral solution (e.g., Nourrit et al., 2003; Vereijken, 1991). In the second category the aim is to understand how a previously learned skill can efficiently adapt to changing environments. Most experiments dealing with questions relative to variability of practice fell in this second category (e.g., Catalano \& Kleiner, 1984; Lai \& Shea, 1998; Moxley, 1977). In that case, transfer tests are preferred to retention tests. In the first category, a change in coordination is obviously expected: during the first trials, participants exploit a 'novice' coordination mode, and practice allows a transition towards a more efficient behavioral organization (Nourrit et al., 2003). This qualitative change in coordination is likely to be revealed in relevant coordination variables. In the second category, a qualitative change in coordination is not really expected. Participants have to adapt an available coordination mode (or a generalized motor program, in the cognitivist framework), and one expects, primarily, an improvement of performance variables (Schmidt, 1982).

Moreover, performance and coordination variables present different dynamics during learning: Nourrit et al. (2003) showed in an experiment on a ski simulator that observed that performance variables (i.e. amplitude and frequency of oscillations) presented a very fast and precocious improvement during practice. In contrast, changes in coordination variables were delayed and occurred only after a prolonged practice time. This kind of result questions the true nature of learning and, obviously, the relevancy of the retention tests that are supposed to check the permanency of changes. In this kind of experiments, learning is conceived as the acquisition of a novel skill, and is essentially revealed by the dynamics of coordination variables. Performance variables are obviously linked to coordination variables (better coordination modes resulting in better performances), but the causal relationship is not equivocal. Performance variable are likely to be directly affected by factors such as motivation, boring, etc. In some tasks, high performance levels can be reached with poor 
behavioral coordination solutions. Finally, a change in coordination can sometimes result in a transitory decrement in performance variables.

Our interest in the present paper focuses on retention, and especially long-term retention. As previously evoked, retention represents one of the major tests for asserting that learning occurred. However, one could argue that retention tests should be performed on coordination variables, rather than on performance variables, of a valid assessment of learning (Nourrit et al., 2003).

Another important point is the delay that could be thought as necessary for testing the permanent character of the changes that occurred during learning. Generally retention tests are conducted following retention intervals ranging from some minutes to some months. The information provided by retention tests differ in nature according to the delay after which they are conducted. When the delay is short, retention tests generally aim at controlling for the effects of experimental factors (augmented feedback, practice schedule, etc.). Longer delays aim a testing the persistence of learning in memory.

Some experiments have tried to assess long-term retention in motor learning (Ammons, Farr, Bloch, Neumann, Mukul, Ralph \& Ammons, 1958; Bell, 1950; Fleishman \& Parker, 1962; Koonce, Chamblis \& Irion, 1964; Hill, 1934, 1957; Neumann \& Ammons, 1957). In these studies, retention delays were generally comprised between 1 and 5 years. A notable exception concerns Hill's studies (Hill, 1934, 1957) where a participant was tested for retention 25 and 50 years, respectively, after learning in typewriting. The use of long-term delays opens different perspectives: after some months or even years, cognitive and physical capacities could obviously be modified (advancing age, weight gain, etc.). Moreover, life offers multiple occasions of learning experiences, of leisure or professional practices that could eventually interfere, positively or negatively, with previous learning. Such retention 
tests aim at evaluating the strength of the traces of learning, and their resistance facing interferences and perturbations.

Most often, these studies evidenced a decrement of performance, but also a quick regain, after some practice (Bell, 1950; Neumann \& Ammons, 1957). The loss of proficiency was proven to depend on various factors, including the nature of the task, the length of the retention interval, the level of proficiency achieved, and the practice of interfering activities during the retention interval (Fleishman \& Parker, 1962; Schendel, Shields, \& Katz, 1978). Note, however, that these studies generally considered rather simple sensory-motor tasks (e.g., rotary pursuit task), and the assessment of retention was exclusively based on performance variables, as previously defined.

In the present study, we analyze long-term retention in a complex motor task, performed on a ski simulator. This apparatus allows participants to perform slalom-like cyclical movements, and was used in a number of experiments, mainly devoted to the analysis of the evolution of motor coordination and performance with practice (Durand, Geoffroi, Varray \& Prefaut, 1994; Nourrit et al., 2003; Vereijken \& Whiting, 1990; Vereijken, 1991; Vereijken et al., 1997; Wulf, Höß \& Prinz, 1998; Wulf \& Weigelt, 1997). We propose a follow-up study of a learning experiment on the ski simulator that was conducted in 2000 in the University of Montpellier (Nourrit et al., 2003). During this experiment five participants practiced on the ski simulator during 39 sessions of ten 1-min trials. The aim of this study was to examine the qualitative behavioral reorganizations that occurred during the acquisition of a complex motor skill. The essential results of this experiment can be summarized as follows. The authors tried to account for coordination through the analysis of the oscillations of the apparatus platform. Using the W-method proposed by Beek \& Beek (1988), they derived from each trial a dynamical model, characterized by distinctive stiffness and damping functions. The results showed that learning could be described as the transition from a "novice behavior", 
characterized by a Rayleigh damping function, to a "skilled behavior", characterized by a van der Pol damping function. More precisely, this experiment showed that learning could be described as the succession of three distinct stages, which were systematically observed for all participants.

During the first stage, participants exploited and stabilized the novice behavior. This stage extended over a large number of trials (from 50 to 150 trials, according to participants). A second stage was characterized by frequent alternations between the novice and the skilled behaviors, from one trial to the next, and also within one trial from one cycle to the next. This second stage also extended over a large number of trials (from 50 to 150 trials, according to participants). Finally, the third stage was characterized by the exclusive exploitation and the stabilization of the skilled behavior.

The authors concluded that the nature of the damping function represented a relevant coordination variable for revealing the evolution of behavior with learning.

The analysis of performance variables (amplitude and frequency) revealed different dynamics: amplitude increased very early with practice and the highest amplitudes were reached during the initial stage. Further practice did not result in a significant increase in amplitude. Oscillation frequency increased suddenly at the beginning of the second stage, and remained then stable up to the end of the experiment.

A first retention test was conducted 5 months after the end of the practice sessions (Deschamps, Nourrit, Caillou, \& Delignières, 2004). Four participants of the initial study were available to participate. Results showed that participants adopted, spontaneously, the van der Pol damping behavior they learned 5 months before, and were able to reach similar amplitudes and frequencies than at the end of the learning sessions. In other words, after 5 months, both performance and coordination variables remained stable, asserting for the persistent character of the changes that occurred during the learning sessions. 
We report in the present paper the results of a second retention test, conducted 10 years after the completion of the initial experiment. The five participants were available and accepted to participate. We hypothesized to observe a significant decrement in performance variables, (i.e. amplitude and frequency). In contrast, we hypothesized that learning led to a deep and stable inscription of the learned coordination mode in the behavioral repertoire of the participants, and that coordination variables should exhibit persistent values, even after this very long delay.

\section{Method}

\subsection{Participants}

The five participants involved in the learning experiment by Nourrit et al. (2003) volunteered to participate in this retention test (four males and one female, mean age: 39.2 years \pm 6.3 , mean weight: $73.2 \mathrm{~kg} \pm 8.46$; mean height $179.6 \mathrm{~cm} \pm 3.5)$. Four of them were occasional skiers (with on average four days of practice per year), but none had training on the ski-simulator since the first experiment. None of them reported serious injuries or diseases during the last decade. Their mean weight gain since the initial experiment was of about 1.6 $\mathrm{kg} \pm$ 8.46. Participants signed a consent form, and were not paid for their participation.

\subsection{Experimental device}

The task was performed on a ski-simulator (Skier's Edge Co., Park City, UT) which consisted of a platform on wheels which moved back and forth on two bowed, parallel metal rails (Figure 1). We used the same modified version of the simulator that replaced the two independent feet supports of the original apparatus with a $30-\mathrm{cm}$ wide board, in unstable balance over a sagittal rotation axis (for more details see Nourrit et al. 2003).

\section{Insert Figure 1}

\subsection{Procedure}


Participants were instructed to make cyclical sideways movements on the ski simulator, "as ample and frequent as possible". They had to keep their hands behind their back at all times, and to fix their eyes on a point located on the floor, three meters in front of the apparatus. They performed a one session of ten 1-min trials with a 1-min break between trials.

\subsection{Data collection}

A passive marker was fixed in the front of the simulator platform. The displacement of this marker was recorded in three dimensions by a VICON motion analyzer (Biometrics) with seven cameras $(100 \mathrm{~Hz})$. Data were collected over 30 seconds, namely from the 15 th to the 45th second of each trial. Analyses focused on the series of positions of the platform, along the transverse axis, computed from the collected 3-D data.

The position time series were filtered with a dual-pass Butterworth filter with a cut off frequency of $10 \mathrm{~Hz}$. We first computed from these series two performance variables: Amplitude (in centimeters), defined as the mean of the maximal deviations of the platform from the central position, at the right and left reversal points of the cycle, and frequency (in Hertz), defined as the inverse of the average time between two successive right reversals.

Each series was then summarized in a normalized average cycle (Mottet \& Bootsma, 1999; Nourrit et al., 2003). First, each series was segmented into half-cycles representing the motion from a reversal point to the following. Each half-cycle was then normalized using 40 equidistant points, by means of linear interpolation. These points were then rescaled within the interval $[-1,+1]$. The normalized half cycles beginning at the same reversal point were averaged point-by-point, and the normalized average cycle (80 points) was obtained by combining the back and forth normalized average half-cycles. The first and second derivatives were computed from the normalized average cycle, and then rescaled to the interval $[-1,+1]$.

The dynamical properties of the oscillations were assessed by means of W-method (Beek \& Beek, 1988; Delignières et al., 1999; Mottet \& Bootsma, 1999; Nourrit et al., 2003; 
Deschamps et al., 2004). This procedure aims at deriving from experimental data a secondorder differential equation of the kind:

$$
\mathrm{m} \ddot{x}+\mathrm{g}(x)+\mathrm{f}(x, \dot{x}) \dot{x}=0
$$

where $x$ represents position. In this equation $\mathrm{g}(x)$ is the stiffness function and $\mathrm{f}(x, \dot{x}) \dot{x}$ the damping function. Beek and Beek (1988) showed that the stiffness and the damping functions were necessarily composed of terms $x^{\mathrm{p}} \dot{x}^{\mathrm{q}}$ (p, q: $0,1,2,3 \ldots$ ), and that a limited catalogue of such terms represented viable transformations of the harmonic oscillator $(\ddot{x}+x=0)$. More precisely, they showed that the stiffness function should be composed of terms from the Duffing series $\left(x^{1}, x^{3}, x^{5}, \ldots\right)$, and the damping function of terms from the van der Pol series $\left(x^{0} \dot{x}, x^{2} \dot{x}, x^{4} \dot{x}, \ldots\right)$ and/or from the Rayleigh series $\left(\dot{x}^{1}, \dot{x}^{3}, \dot{x}^{5}, \ldots\right)$, separately or in combination.

We used the simple graphical methods proposed by Beek \& Beek (1988) to settle on the terms to include in the model. In a first step, the examination of Hooke's portraits (acceleration vs position) allowed a rather direct identification of the terms composing the stiffness function. In order to determine the non-linear damping terms, The authors proposed to perform a regression of - $\ddot{x}$ against all previously identified linear and non-linear stiffness terms, and linear damping $(\dot{x})$. The residual $(R E S)$ of this regression is assumed to reflect the contribution of non-linear damping terms on behavior. Then it is possible to seek for a van der Pol behavior by plotting $R E S / \dot{x}$ as a function of $x$ (in this case a parabola is expected, revealing the presence of a $x^{2} \dot{x}$ term in the residuals), and for a Rayleigh behavior by plotting $R E S$ as a function of $\dot{x}$ (expecting an $\mathrm{N}$ shape, evidencing the presence of a $\dot{x}^{3}$ term in the residuals). This procedure allows providing a first minimal model. On that basis, the respective weights of each term can then be determined by a stepwise multiple regression procedure onto - $\ddot{x}$ (see Nourrit et al., 2003 for details). 
Nourrit et al. (2003) showed that the initial behavior adopted by beginners participants on the ski simulator could be modeled by a strongly nonlinear stiffness function including cubic and quintic Duffing terms, and a Rayleigh damping function:

$$
\ddot{x}+\mathrm{c}_{10} x+\mathrm{c}_{30} x^{3}+\mathrm{c}_{50} x^{5}+\mathrm{c}_{01} \dot{x}+\mathrm{c}_{03} \dot{x}^{3}=0
$$

Note that according to the $\mathrm{W}$-method notation, $\mathrm{c}_{\mathrm{ij}}$ denote the coefficient associated with the term $x^{\mathrm{i}} \dot{x}^{\mathrm{j}}$

In contrast, the skilled behavior, stabilized by a large amount of practice ( 39 sessions of ten 1-min trials), included a van der Pol damping function with a nonlinear damping term $x^{2} \dot{x}$

$$
\ddot{x}+\mathrm{c}_{10} x+\mathrm{c}_{30} x^{3}+\mathrm{c}_{50} x^{5}+\mathrm{c}_{01} \dot{x}+\mathrm{c}_{21} x^{2} \dot{x}=0
$$

Note that the viability of these models imposes specific sign constraints, which represent a final test for their relevancy: in all cases the linear damping term $\mathrm{c}_{01}$ must be negative and the nonlinear damping term $\left(\mathrm{c}_{03}\right.$ or $\left.\mathrm{c}_{21}\right)$ positive, for the limit cycle dynamics sustainability.

\section{Results}

For all variables we presented the values obtained for each participant and each trial. For a comparison with the previous experiment (Nourrit et al., 2003), we also reported the corresponding values for the 21 th, $100^{\text {th }}$, and $395^{\text {th }}$ trials of the practice sessions. At this point, it is important to remind that we did not consider the estimates obtained during the two first practice sessions (trials 1 to 20), characterized by a particularly erratic and irregular behavior. Thus the $21^{\text {th }}$ trial was considered as a relevant example of the stabilization of the novice behavior during the initial learning stage. The $100^{\text {th }}$ trial was selected because it corresponded for all participants to the transition stage. Finally, the $385^{\text {th }}$ trial was typical of final stabilization of the skilled behavior observed in the last practice session.

\subsection{Amplitude}

Insert Figure 2 
During the first trial of the retention test, participants reached a mean amplitude of $25.22 \mathrm{~cm}$ $(S D=6.41)$, revealing a decrement of $32.0 \%$ with respect of the amplitudes reached during the last trials of the initial experiment. Amplitude tended to increase slightly over the 10 trials, up to a mean value of $30.87 \mathrm{~cm}(S D=2.82)$ for the last trial. As explained in the introduction, amplitude increased very early during the initial experiment, and the mean amplitude during the 21th trial was already about $30.09 \mathrm{~cm}(S D=6.07)$. As a reference, during the very first trial participants reached a mean amplitude of $9.92 \mathrm{~cm}(S D=8.83)$.

\subsection{Frequency}

\section{Insert Figure 3}

During the first trial of the retention test, mean frequency was of $1.14 \mathrm{~Hz}(\mathrm{SD}=0.16)$, corresponding to a decrease of $20.4 \%$ with respect of the mean frequency observed at the end of the previous experiment. Mean frequency increased slightly over the ten trials, up to 1.26 $\mathrm{Hz}(S D=0.14)$ during the last trial. At the end of the retention test, mean frequency remained below that observed during the transition stage of the initial experiment (e.g., $100^{\text {th }}$ trial, $1.29 \mathrm{~Hz}, S D=0.15)$.

\subsection{Limit cycle modeling}

We used the graphical tools proposed by Beek \& Beek (1988) for determining the terms to include in a minimal model accounting for our data. We first analyzed the Hooke's portraits (acceleration vs position), in order to settle on the terms to include in the stiffness function. We present in Figure 4 an illustrative example of the individual Hooke's portraits. With respect to the theoretical harmonic trend $(\ddot{x}=x)$, one can observe a typical slowing down when $x$ is moved from the resting position, and a restoration of stiffness when approaching the reversal points of the movement. The first phenomenon can be accounted for by a negative cubic Duffing term $-x^{3}$ ( Mottet \& Botsma, 1999), and the second by a positive quintic term $x^{5}$, (Nourrit et al., 2003). 


\section{Insert Figure 4}

In accordance with our hypotheses, we sought for the presence of van der Pol damping in our series. Applying the method proposed by Beek \& Beek (1988), we computed for each trial the residuals $(R E S)$ of the regression of the identified stiffness terms $\left(x, x^{3}\right.$, and $\left.x^{5}\right)$, and the linear damping term $(\dot{x})$ onto $-\ddot{x}$. Then we plotted $R E S / \dot{x}$ against $x$, seeking for a parabola revealing the presence of a two-order van der Pol term $\left(x^{2} \dot{x}\right)$ in the damping function. We present in Figure 5 example plots (Participant 2, trials 1, 5 and 10): in all cases we observed the expected U-shape. We also plotted RES against $\dot{x}$ for checking for the presence of a Rayleigh behavior, but we never found any trace of the expected $\mathrm{N}$-shape.

\section{Insert Figure 5}

Thus we performed a multiple regression for estimating the coefficients of the resulting Duffing-van der Pol model (Eq. 3). As an example, we present in Table 1 the estimates obtained for each participant, for the sixth trial. In all cases the models satisfied signs requirements, $c_{01}$ being negative and $c_{21}$ positive, with $r^{2}$ ranging from 0.89 to 0.97 . Similar results were obtained for all trials. We present in Figure 6 the $c_{01}$ coefficients (linear damping), which were negative in all cases. These results clearly showed that all participants adopted, during the 10 trials of the retention test, a damping behavior similar to that observed at the end of the initial experiment.

\section{Insert Table 1}

\subsection{Evolution of the stiffness function}

We present in Figure 6 the evolution of the nonlinear stiffness coefficient $c_{30}$. We limited our analyses to $c_{30}$ because the coefficients of the stiffness function tend to strongly covary in absolute values, $c_{10}$ and $c_{50}$ being positive and $c_{30}$ negative. The linearization of the stiffness function is revealed by $\mathrm{c}_{30}$ values close to zero. 
During the first trials of the initial experiment, the nonlinear stiffness coefficient $c_{30}$ was very low indicating strong nonlinearities in the stiffness function. These nonlinearities tended to disappear from the beginning of the transition stage, as revealed by the values reported for the $100^{\text {th }}$ and the $385^{\text {th }}$ trials. With respect to the values observed at the end of the practice sessions, which were centered around zero, the nonlinear stiffness coefficient $\mathrm{c}_{30}$ was mainly negative during this current retention test, indicating a resurgence of nonlinearities in the stiffness function. During the $2^{\text {nd }}$ trial, the mean value was $-1.55(\mathrm{SD}=1.15), \mathrm{c}_{30}$ then tended to progressively increase over trials, and finally the mean value was $-0.29(\mathrm{SD}=0.81)$ at the end of the retention test, evidencing a progressive linearization of the stiffness function over the 10 trials.

\section{Insert Figure 6}

\subsection{Evolution of the damping function}

In order to account for the dynamics of learning through a unique metrics, despite the presence of qualitative transition from a Rayleigh to a van der Pol damping behavior, Nourrit et al. (2003) proposed to force the Duffing + Rayleigh model (Eq. 2) to all series. They showed that the obtained linear damping coefficient $\mathrm{c}_{01 \text { (Rayleigh) }}$ took on negative values when the limit cycle was supported by a Rayleigh damping behavior, and conversely positive values when the limit cycle was supported by a van der Pol damping function. In this second case, the forced linear damping coefficient $\mathrm{c}_{01(\text { Rayleigh) }}$ was roughly proportional, in absolute value, to the $\mathrm{c}_{01}$ coefficient computed with the Duffing + van der Pol model (Eq. 3). As such, the transition from the novice to the skilled behavior can be described as an evolution of $\mathrm{c}_{01(\text { Rayleigh) }}$ from negative to positive values (Nourrit et al., 2003). In order to compare the results of this retention test with those of the initial experiment, we computed this forced $\mathrm{c}_{01 \text { (Rayleigh) }}$ for each trial (Figure 7). 
As expected, the $\mathrm{c}_{01 \text { (Rayleigh) }}$ presented positive values, for all participants and all retention trials. This result clearly indicates that all participants, right from the first trial, adopted the van der Pol damping behavior then learned and highly stabilized 10 years ago. Moreover, this forced linear damping coefficient $\mathrm{c}_{01 \text { (Rayleigh) }}$ tended to increase over the ten trials, from a mean value of $0.52(\mathrm{SD}=0.40)$ for the first trial, to a mean value of $1.00(\mathrm{SD}=0.26)$ for the last trial. These last values are slightly higher than those reported at the end of the previous experiment, suggesting the strong stability of the van der Pol damping behavior, despite the very long no-practice period.

\section{Insert Figure 7}

\section{Discussion}

The main result of the present experiment is the persistence of the acquired coordination mode, ten years after the learning sessions. Ten years ago, the five participants practiced for a long time for overcoming their initial behavior on the task, and for adopting and stabilizing the skilled behavior characterized by a van der Pol form of damping in the oscillations of the ski simulator. Ten years after, all participants spontaneously adopted from the first trial this skilled behavior (see figure 6). This behavior is conceived as the signature of "expert" (skilled) coordination mode (Nourrit et al., 2003), and its exploitation confirms that motor learning is characterized by persistent changes in behavior over time (Newell et al., 2001). The continuous motor skill (as oscillations in ski-simulator) is generally conceived more persistent than discrete procedural response for example and the relearning after a retention interval is more rapid than the previous learning (Schendel et al., 1978). Some general principles in long-term retention are known since a long time, but this topic of long-term motor retention is few investigate and suggests a few productive ideas (Adams, 1987). Interestingly, this persistence of coordination mode is consistent with the conceptions developed by the proponents of the dynamical systems approach, considering the acquisition 
of a new skill as stabilizing a new attractor in the repertoire of the organism (Zanone \& Kelso, 1992; 1997): any transformation in the attractors landscape is "catastrophic" in the sense of Thom (1983), and the phase space is modified in an irreversible way. Importantly, the $\mathrm{C}_{01 \text { (Rayleigh) }}$ parameter tended to progressively increase along the ten trials of the retention test, suggesting a fast enhancement of the stability of the van der Pol damping behavior, just after some minutes of practice. So we can confirm that the persistent change arises from stability of attractor landscape (Newell et al., 2001; Newell, Mayer-Kress, Hong, \& Liu, 2009).

Concerning amplitude and frequency, in contrast, we observed a clear performance decrement, with respect to the values reported at the end of the initial experiment. Amplitude decreased by about $18 \%$, and frequency by about $13 \%$. During the ten trials of the retention test, a slight increase in amplitude and frequency was observed, on average, but participants never reached amplitudes or frequency values similar to those reported at the end of the initial experiment. This result is interesting, because a number of previous experiments on long-term retention also described a decrement in performance during the first trials, but a fast regain, at least when compared with the time it took for reaching, during the initial learning, the skilled level of performance. Typically, subjects show a reduction in performance of just $20 \%$ and regain rapidly their previous skill after few practice trials (Bell, 1950; Neumann \& Ammons, 1957, Willingham \& Dumas, 1997).

We don't observe, in the present experiment, any evidence for a fast reimprovement in performance: despite a cumulative practice of ten minutes, an increase in amplitude was moderate, especially beyond the fourth trial, and frequency remained stable over the ten trials. One have to keep in mind that in contrast with most tasks previously tested for long-term retention, the ski-simulator is physically highly demanding (Durand, Geoffroy, Varray \& Préfaut, 1994; Teulier, Nourrit \& Delignières, 2006). Oscillating at large amplitude and high frequency requires an important energy expenditure. Ten years ago, four of the participants 
were student in the Faculty of Sport Sciences of Montpellier, and were obviously in good physical condition. Ten years after, they were less physically active, and even if their mean weight gain was negligible, their physical capacities were lower than during the initial experiment.

The low oscillation frequencies observed in the present experiment raise an interesting question. During the initial experiment, a sudden increase in frequency was observed in all participants, from about $1 \mathrm{~Hz}$ to $1.4 \mathrm{~Hz}$, and this abrupt change in frequency was concomitant, individually, with the beginning of the transition stage, during which participants used the novice and skilled coordination modes in alternation. According to Nourrit et al. (2003), frequency could be considered a control parameter, favoring beyond a given threshold the availability of the skilled coordination mode. Interestingly, during the present experiment participants exploited spontaneously the skilled coordination mode, while oscillating at frequencies clearly below the threshold that was supposed to favor the availability of the van der Pol damping. This showed that the increase in frequency, during learning, was necessary for exploring the workspace and discovering the skilled coordination mode. However, once learned the skilled coordination mode appears independent on frequency and could be exploited even with slow oscillations. In this regard, the question of control parameter identification for this skill is raised again.

The evolution of the stiffness function gave more ambiguous results. We only reported results for the cubic Duffing term $\mathrm{C}_{30}$, but these results gave a good image of the nonlinearity of stiffness. During the previous experiment, the initial stage was characterized by a strongly nonlinear stiffness function. Nourrit et al. (2003) suggested that this nonlinear function , and essentially the cubic Duffing term, provided participants with a kind of local dwelling time, allowing to manage more easily the reversal points of the oscillations of the platform (for similar arguments see Mottet \& Bootsma, 1999). The present results showed that, at least 
during the first trials of the retention test, participants tended to exploit such nonlinearities. Most of them, however, recovered from the fifth trial the values reported at the end of the initial experiment. These results indicate a loss of behavior stability when the subjects retake the practice: behavior is persistent but just instable. This confirms that the observed changes exhibit both persistent and transitory properties (Newell et al., 2001) and depend on studied time scales. Even if the skilled behaviour is long-lasting, a period of rest makes this behavior less stable.

The main message of the present study is the very different image of retention that provide performance and coordination variables. On the basis of the former, one could conclude on a very poor retention over time. The latter in contrast revealed the strong persistence of acquired coordination modes. The concept of coordination variable is close to that of essential variable introduced by Gel'fand \& Tsetlin (1962, see also Vereijken, 1991). Essential variables reflect the behavioral structure of coordination modes, in terms of topological quality. In contrast, inessential variables (or nonessential variables for Vereijken, 1991) inform about scaling changes within a given coordination mode, providing the system with flexibility. From this point of view, the essential variable, in the present context, is the nature of the dynamical model that provides the best account for the dynamics of the motion of the platform, i.e. the Duffing + Rayleigh model (Eq. 2), or the Duffing + van der Pol model (Eq. 3), considering that these models differs only by the presence of a nonlinear damping term $\dot{x}^{3}$ in the first case, and $x^{2} \dot{x}$ in the second. The coefficient $\mathrm{C}_{01 \text { (Rayleigh) }}$ is a statistical tool that allows to distinguish these two qualitatively distinct models by means a similar metrics. Note that this coefficient can be considered both an essential and an inessential variable. The essential variable is the sign of this coefficient: negative and positive values are associated with qualitatively distinct coordination modes, novice and skilled, respectively. The absolute

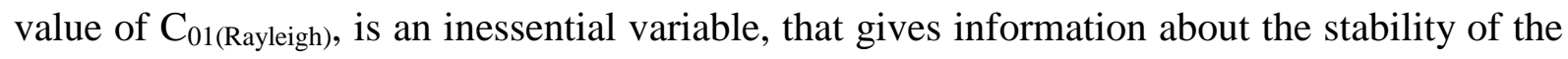


coordination mode. As well, the values of the coefficients of stiffness function are inessential variables

According to the definition proposed by Gel'fand \&Tsetlin (1962), performance variables appear a neither essential, nor inessential: they just describe the outcomes of behavior, and the present results showed that distinct coordination modes could yield similar performance levels.

\section{Conclusion}

It seems clear that the choice of relevant variables is essential for providing efficient tests for learning and retention. With this regard, performance variables, frequently used in learning experiments, represent rather poor indicators. Performance is known to be affected by a number of factors, beyond skill level, including motivation, self-confidence, expectancies, and physical condition. This experiment suggests that these factors could sometimes be dominant in the determination of performance, and completely hide the effects of learning. Coordination variables offer a valuable alternative, and the present results show that acquired coordination modes persist still for a long time after learning, supporting the popular motto: "Once one learns how to ride a bicycle, one never forgets how to do so".

\section{Acknowledgments}

The authors would like to thank Euromov, the Technological Platform of Montpellier 1, that allowed us to use the experimental materials (ski simulator) and the Vicon Motion Analyzer (Biometrics) for the data collection.

\section{References}

Abernethy, B. \& Sparrow, W.A. (1992). The rise and fall of dominant paradigms in motor behaviour research. Dominant paradigms in motor behaviour. In Summers, J.J. (ed). 
Approaches to study of motor control and learning (pp. 3-45). Amsterdam: Advances in Psychology, 84, North-Holland, Chap 1, pp 3-45.

Adams, J.A. (1987). Historical review and appraisal of research on learning, retention, and transfer of humaun motor skill. Psychological Rewiew, 101, 41-74.

Ammons, R.B., Farr, R.G., Bloch, E. ,Neumann, E., Mukul, D., Ralph, M. \& Ammons, C.H. (1958). Long-term retention of perceptual-motor skills. Journal of Experimental Psychology, 55, 318-328.

Beek, P.J., \& Beek, W.J. (1988). Tools for constructing dynamical models of rhythmic movement. Human Movement Science, 7, 301-342.

Beek, P.J., Peper, C.E. \& Stegeman, D.F. (1995). Dynamical Models of Movement Coordination. Human Movement Science, 14, 573-608.

Bell, H.M. (1950). Retention of pursuit rotor skill after one year. Journal of Experimental Psychology, 55, 318-328.

Catalano, J.F. \& Kleiner, B.M. (1984). Distant transfer in coincident timing as a function of variability of practice. Perceptual and Motor Skills, 58, 851-856.

Delignières, D., Nourrit, D. Deschamps, T., Lauriot, B. \& Caillou, N. (1999). Effects of practise and tasks constraints on stiffness and friction functions in biological movements. Human Movement Science, 18, 769-793.

Delignières, D., Nourrit, D., Sioud, R., Leroyer,P. Zattara, M., \& Micaleff, J-P. (1998). Preferred coordination modes in the first steps of the learning of a complex gymnastics skill. Human Movement Science, 17, 221-241.

Deschamps, T., Nourrit, D., Caillou, N., \& Delignières, D. (2004). Influence of a stressing constraint on stiffness and damping functions of ski simulator's platform motion. Journal of Sport Sciences, 2, 867-874. 
Durand, M., Geoffroi, V., Varray, A., \& Prefaut, C. (1994). Study of the energy correlates in the learning of a complex self-paced ski11. Human Movement Science, 13, 785-799.

Fleishman, E.A., \& Parker, J.F. (1962). Factors in the retention and relearning of perceptualmotor skill. Journal of Experimental Psychology, 64 (3), 215-226.

Gel'fand, I.M., \& Tsetlin, M.L. (1962). Some methods of control for complex systems. Russian Mathematical Surveys, 17, 95-116.

Hill, L.B. (1934). A quarter Century of delayed recall. Pedagogical Seminary, 44, 231-238

Hill, L.B. (1957). A second quarter Century of delayed recall or relearning at 80. The journal of Educational Psychology, 48, 65-68.

Kelso, J. A. S. (1995). Dynamic patterns: The self-organization of brain and behavior. Bradford: MIT Press.

Koonce, J.M., Chambliss, D.J. \& Irion, A.L. (1964). Long term reminiscence in pursuit-rotor habit. Journal of Experimental Psychology, 67 (5), 498-500.

Lai, Q. \& Shea, C.H. (1998). Generalized motor program (GMP) learning : Effects of reduced frequency of knowledge of results and practice variability. Journal of Motor Behavior, 30, $51-59$.

Mottet, D., \& Bootsma, R.J. (1999). The dynamics of goal-directed rhythmical aiming. Biological Cybernetics, 80, 235-245.

Moxley, S.E. (1979). Schema: the variability of practice hypothesis. Journal of Motor Behavior, 11, 65-70.

Neumann, E., \& Ammons, R.B. (1957). Acquisition and long-term retention of a simple serial perceptual-motor skill. Journal of experimental Psychology, Vol 53, n³, 159-161.

Newell, K.M. (1991). Motor Skill Acquisition. Annual Review of Psychology, 42, 213-237.

Newell, K.M., Liu, Y.T., \& Gottfried, M.K. (2001). Times scales in motor learning and developpement. Psychological Review. Vol 108; n¹; 57-82 
Newell, K.M., Mayer-Kress, G., Hong, S.L. \& Liu, Y-T. (2009). Adaptation and learning: characteristic time scales of performance dynamics. Human Movement Science, 28; 655687.

Nourrit, D., Delignières, D., Caillou, N., Deschamps, T., \& Lauriot, B. (2003). On discontinuities in motor learning: A longitudinal study of complex skill acquisition on a ski-simulator. Journal of Motor Behavior, 35, 151-170.

Schendel, J.D., Shields, J.L., \& Katz, M.C. (1978). Retention of motor skill: Review. Research Institute for the Behavioral and Social Sciences, Technical Paper 113.

Schmidt, R.A. (1982). Motor control and learning : a behavioral emphasis. Champaign : Human Kinetics Publishers.

Teulier, C., Nourrit, D. \& Delignières, D. (2006). The evolution of oscillatory behavior during learning on a ski simulator. Research Quarterly for Exercise and Sport. , 77, n³, 464475.

Thom, R. (1983). Paraboles et Catastrophes. Paris: coll.: Champs, ed.: Flammarion, Paris.

Vereijken, B. \& Whiting, H.T.A. (1990). In defence of discovery learning. Canadian Journal of Sport Psychology, 15, 99-106.

Vereijken, B. (1991). The dynamics of skill acquisition. Amsterdam: Free University Press.

Vereijken, B., Van Emmerik, R.E.A., Bongaardt, R. , Beek, W.J., \& Newell, K.M. (1997). Changing coordinative structures in complex skill acquisition. Human Movement Science, $16,823-844$.

Willingham, D.B. \& Dumas, J.A. (1997). Long-term retention of a motor skill: implicit sequence knowledge is not retained after a one-year delay. Psychological Research, 60, 113-119.

Wulf, G. \& Weigelt, C. (1997). Instructions about physical principles in learning a complex motor skill: to tell or not to tell... Research Quarterly for Exercise and Sport, 68, 362-367 
Wulf, G., Höß, M., \& Prinz, W. (1998). Instructions for motor learning: Differential effects of internal versus external focus of attention. Journal of Motor Behavior, 30, 169-179.

Zanone, P.G., \& Kelso, J.A.S. (1992). Evolution of behavioral attractors with learning: nonequilibrium phase transitions. Journal of Experimental Psychology: Human Perception and Performance, 18, 403-421.

Zanone, P.G., \& Kelso, J.A.S. (1997). Coordination dynamics of learning and transfer: collective and component levels. Journal of experimental Psychology: Human Perception and Performance, 23, 1454-1480. 


\section{Figure Captions}

Figure 1: The ski simulator

Figure 2: Evolution of the individual mean amplitude, for three selected trials of the 2000's experiment, and for the 10 trials of the retention test.

Figure 3: Evolution of the individual mean frequency, for three selected trials of the 2000's experiment, and for the 10 trials of the retention test.

Figure 4: Representative Hooke's portrait (acceleration vs position, participant 2, trial 10)

Figure 5: Representative plots of RES/ $\dot{x}$ vs $x$ (participant 2, left panel: trial 1, middle panel: trial 5, right panel: trial 10)

Table 1: Estimates of the coefficients of the Duffing + van der Pol model $\left(\ddot{x}+\mathrm{c}_{10} x+\mathrm{c}_{30} x^{3}+\right.$ $\left.\mathrm{c}_{50} x^{5}+\mathrm{c}_{01} \dot{x}+\mathrm{c}_{21} x^{2} \dot{x}=0\right)$, for the sixth trial.

Figure 6: $\mathrm{c}_{10}$ coefficients (linear damping), for each participant and each trial of the retention test.

Figure 7: Evolution of the individual nonlinear stiffness coefficient $c_{30}$, for three selected trials of the 2000's experiment, and for the 10 trials of the retention test.

Figure 8: Evolution of the individual nonlinear damping coefficient $\mathrm{C}_{01 \text { (Rayleigh), for three }}$ selected trials of the 2000's experiment, and for the 10 trials of the current retention test. 


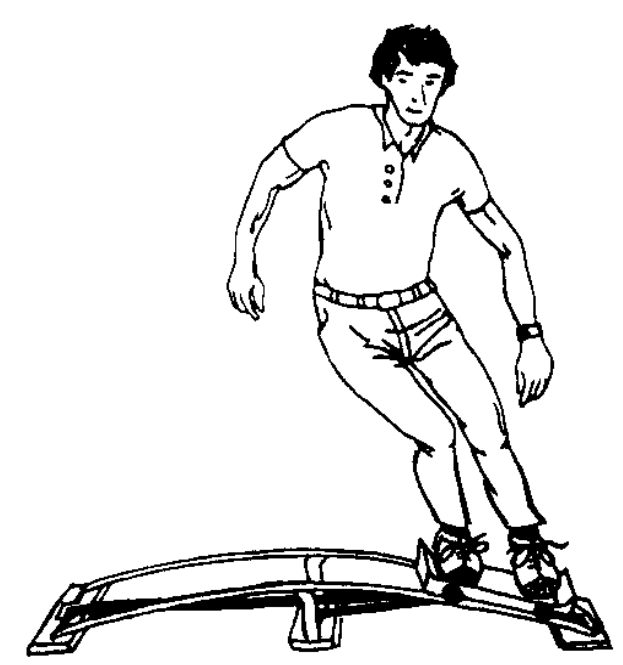

Figure 1 


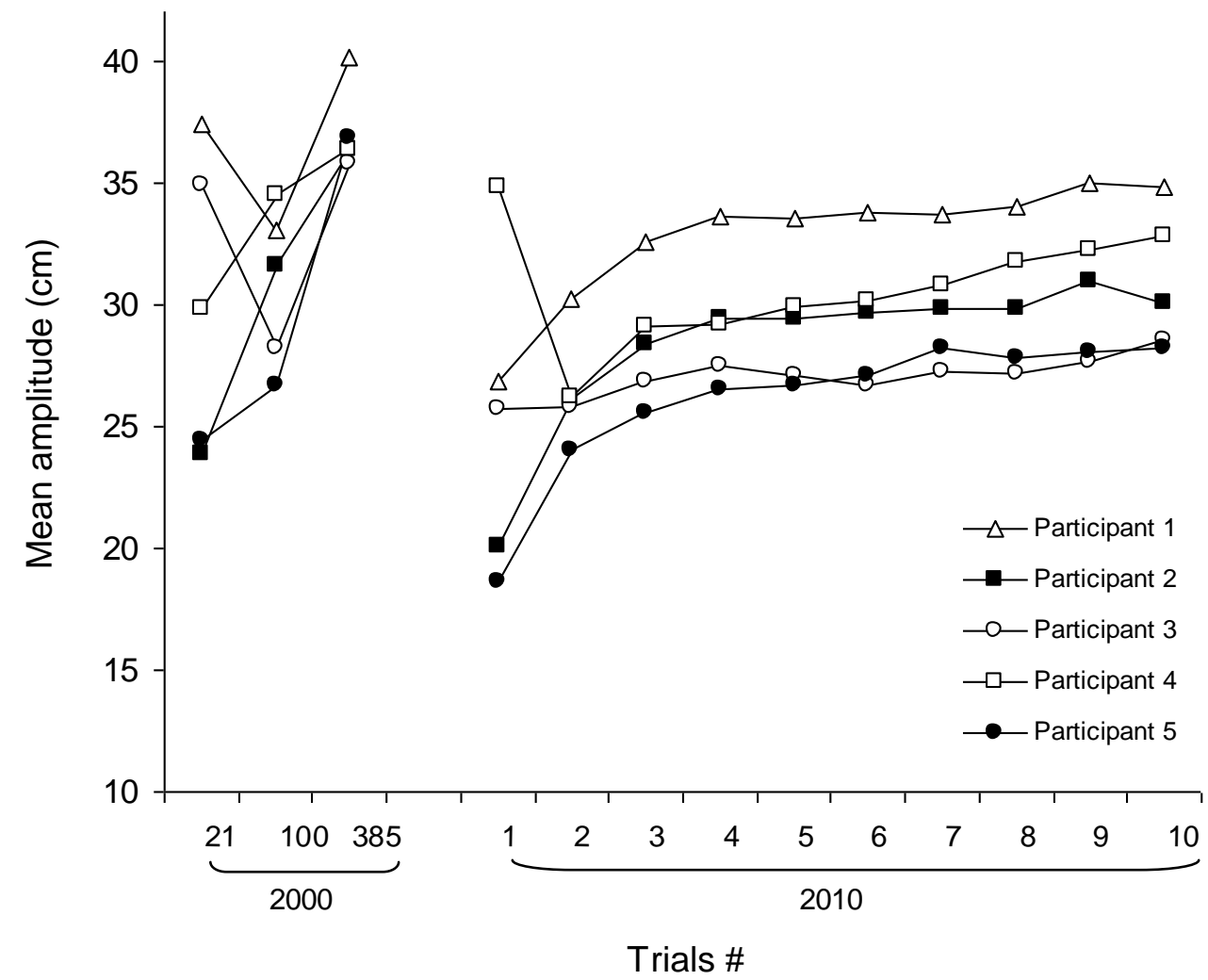

Figure 2 

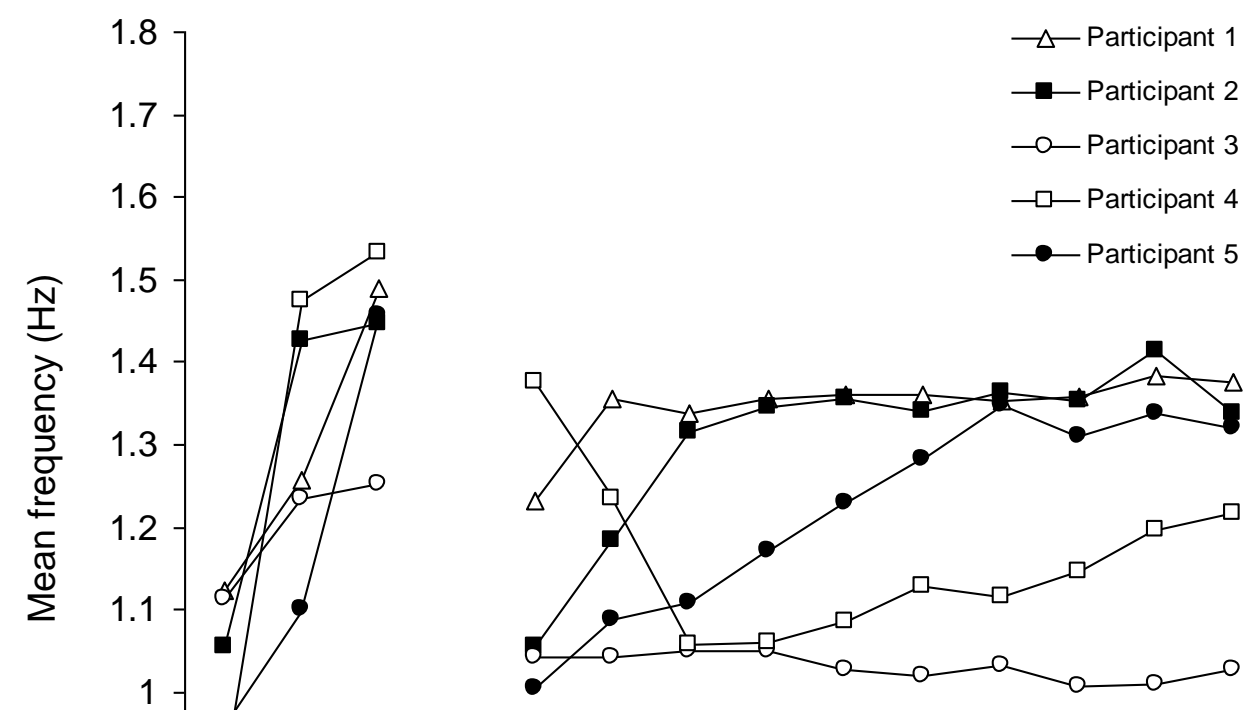

Figure 3

Trials \# 


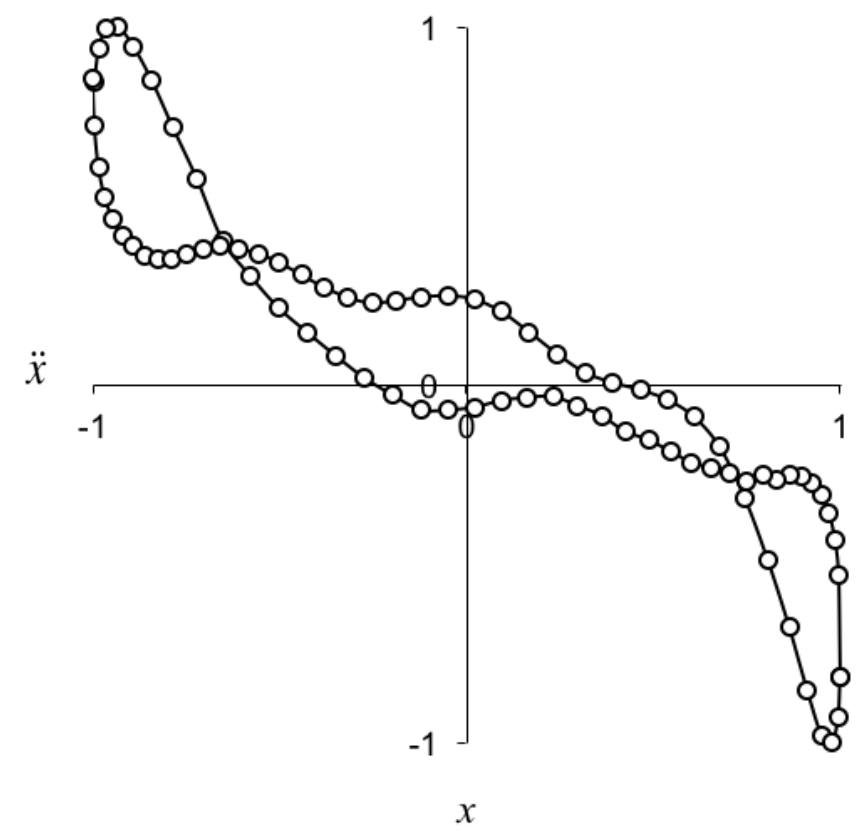

Figure 4 

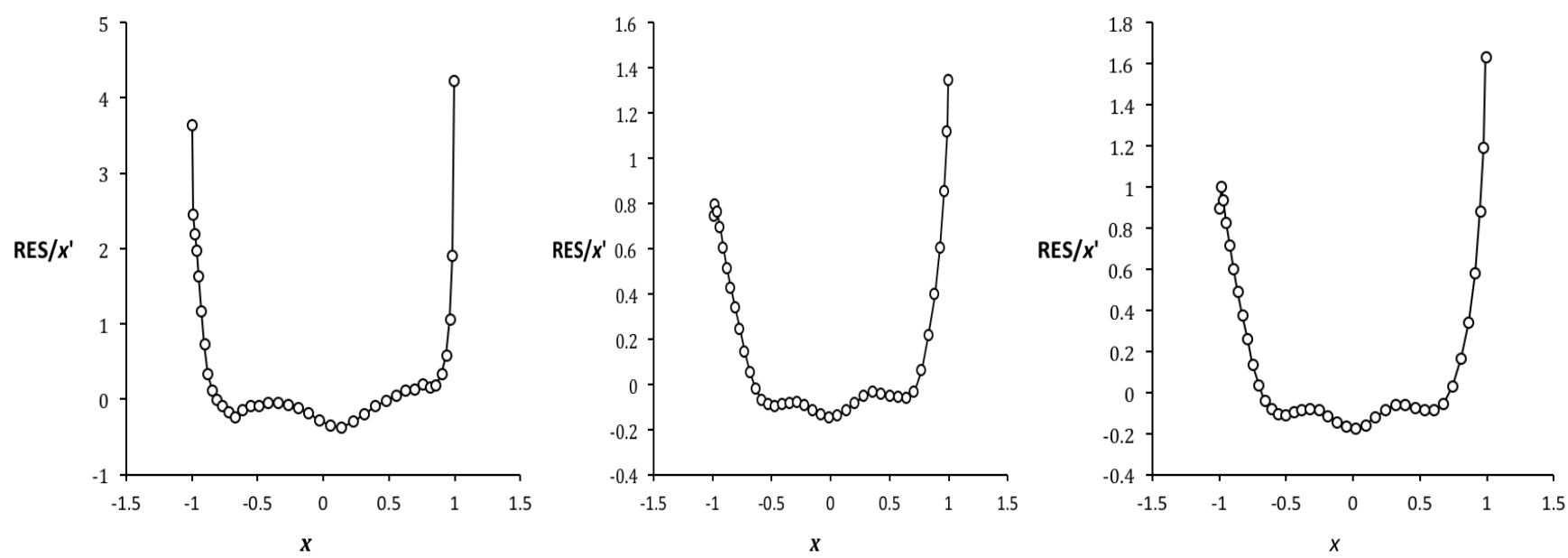

Figure 5 


\begin{tabular}{l|c|c|c|c|c|c}
\hline & $\mathrm{C}_{10}$ & $\mathrm{C}_{30}$ & $\mathrm{C}_{50}$ & $\mathrm{C}_{01}$ & $\mathrm{C}_{21}$ & $\mathrm{r}^{2}$ \\
\hline Participant 1 & 0.96 & -1.13 & 1.14 & -0.23 & 0.32 & 0.89 \\
Participant 2 & 1.00 & -0.70 & 0.65 & -0.30 & 0.43 & 0.94 \\
Participant 3 & 1.19 & -0.87 & 0.67 & -0.17 & 0.24 & 0.94 \\
Participant 4 & 1.49 & -2.50 & 1.96 & -0.19 & 0.28 & 0.83 \\
Participant 5 & 0.58 & -0.03 & 0.42 & -0.27 & 0.37 & 0.97 \\
\hline
\end{tabular}

Table 1 


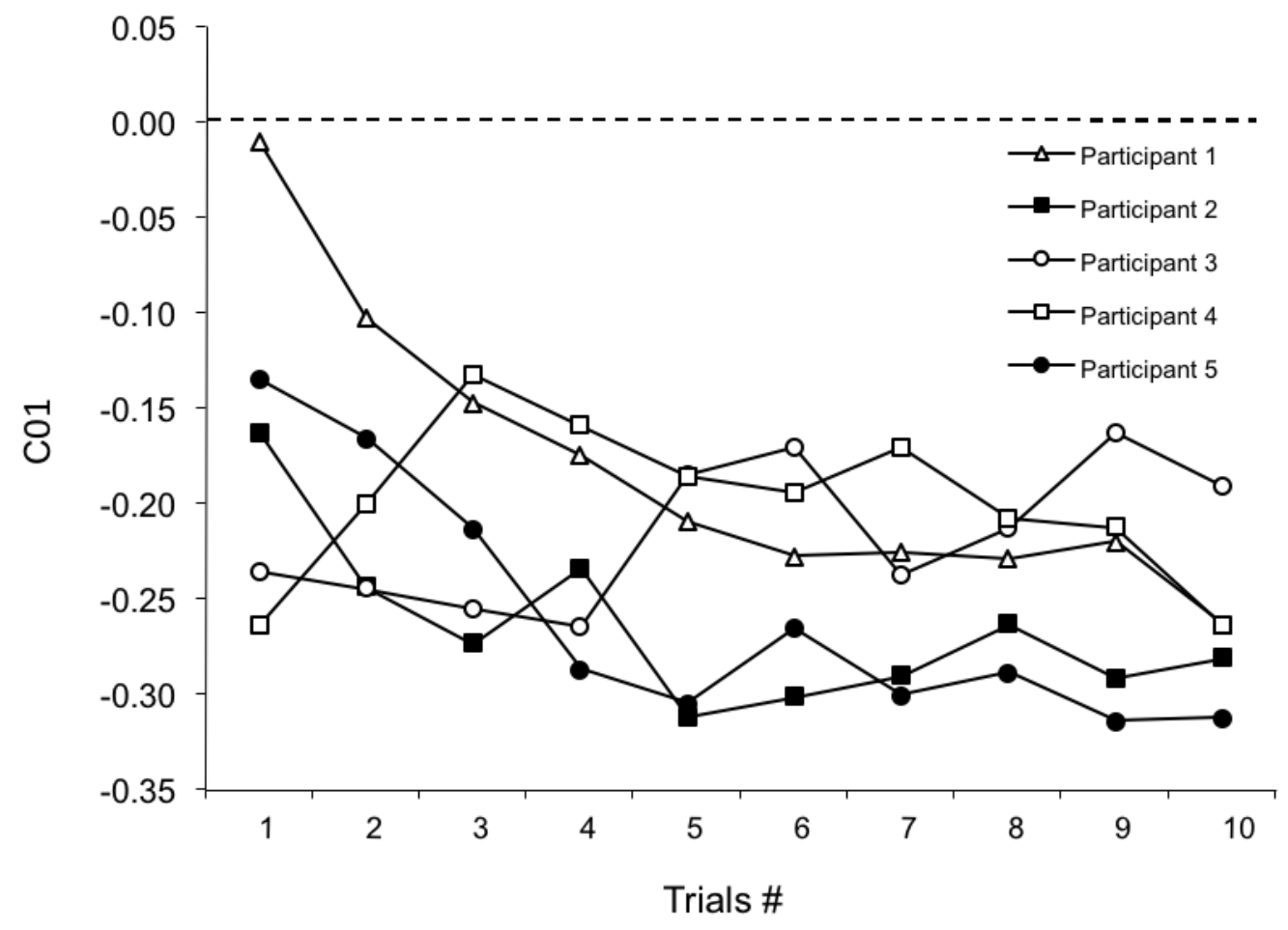

Figure 6 


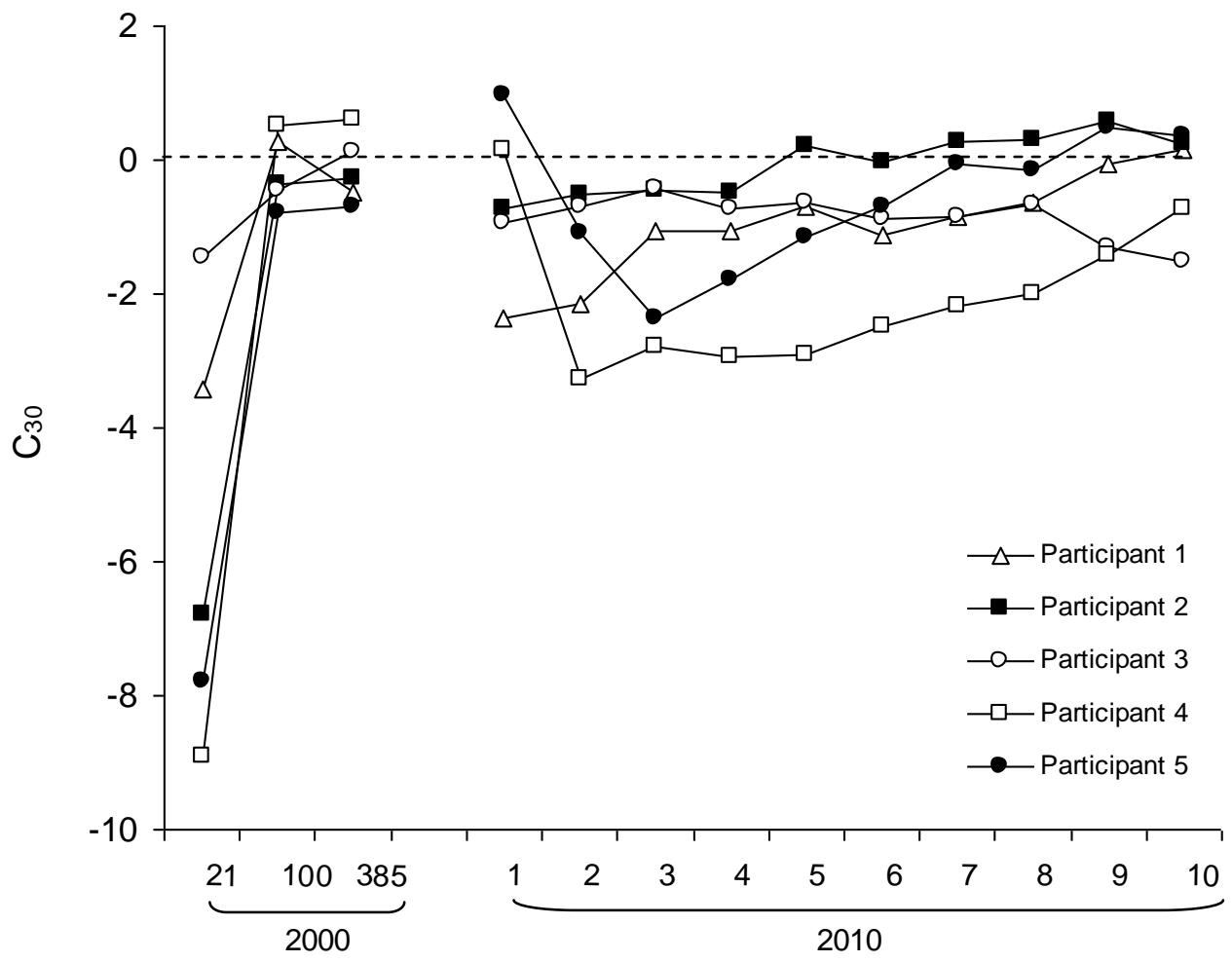

Trials \#

Figure 7 


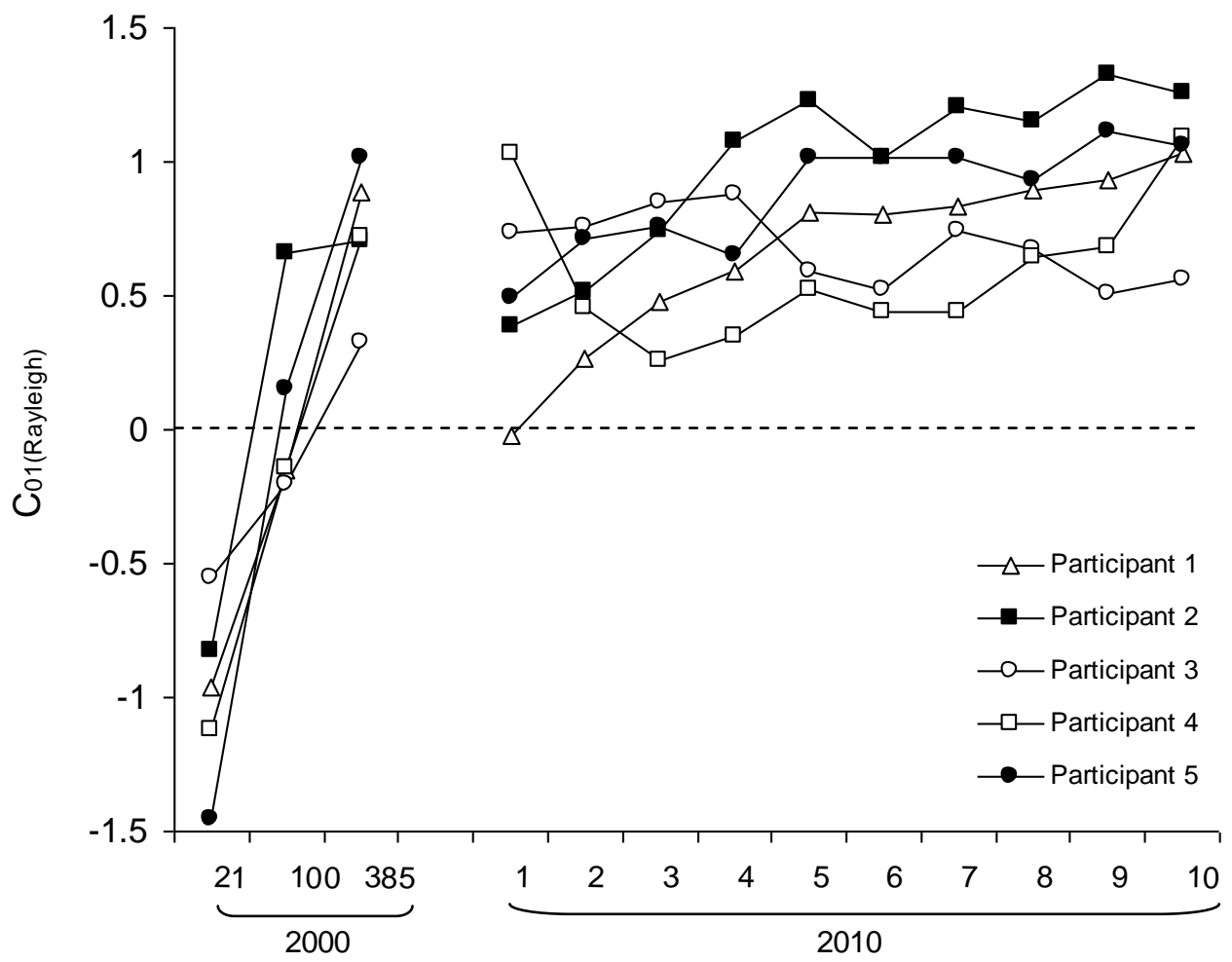

Trials \#

Figure 8 